\title{
Apuntes sobre epistemología e investigación en la enseñanza de los Estudios Sociales
}

\author{
Notes on Epistemology and Research in \\ Social Studies Teaching
}

Luis Carlos Morales Zúñiga

Escuela de Formación Docente de la

Universidad de Costa Rica

San José, Costa Rica

luis23m@gmail.com

Recibido 30-09-2010 • Aceptado 18-10-2010 • Corregido 25-10-2010

\begin{abstract}
Existe una opinión muy generalizada según la cual la matemática es la ciencia más difícil cuando en realidad es la más simple. La causa de esta paradoja reside en el hecho de que, precisamente por su simplicidad, los razonamientos matemáticos equivocados quedan a la vista. En una compleja cuestión de política o arte, hay tantos factores en juego y tantos desconocidos o inaparentes que es muy difícil distinguir lo verdadero de lo falso.
\end{abstract}

Ernesto Sábato

\begin{abstract}
Resumen: Este ensayo analiza el concepto de estudios sociales contrastándolo con la noción conceptual denominada didáctica de las ciencias sociales, esto con el fin de observar las particularidades de cada categoría. Posteriormente, se plantea un esbozo de epistemología aplicada al campo de los Estudios Sociales, con el objetivo de repensar la construcción de objetos de investigación y de conocimiento en este campo. Por último, se reflexiona sobre la posibilidad de establecer orientaciones o lineas de investigación que sistematicen de alguna manera los procesos investigativos en la enseñanza de los estudios sociales.
\end{abstract}

Palabras clave: Estudios sociales, didáctica, epistemología, investigación, educación.

\section{Introducción}

En el epígrafe citado Ernesto Sábato se refiere a la complejidad en la construcción de conocimientos válidos en política y arte, no obstante, es plausible extender esa complejidad al resto de las Ciencias Sociales y Humanas, donde las Ciencias de la Educación resultan ser un campo en el que particularmente se suelen emitir criterios y juicios de aparente validez, aunque estos carezcan de una fundamentación teórica o empírica como base argumentativa. En 


\begin{abstract}
This article examines the concept of social studies, contrasting it with the conceptual notion called didactic of social sciences, in order to observe the particularities of each category. Subsequently a sketch of epistemology applied to the field of social studies is presented, with the goal to rethink the construction of knowledge and research objects in this field. Finally we thought on the possibility of establishing directions or systematic research lines which will organize the investigation processes in the teaching of social studies.
\end{abstract}

Key words: Social studies, didactic, epistemology, research, education la Enseñanza de los Estudios Sociales también se genera esa problemática $\mathrm{y}$, por lo tanto, se considera necesario esbozar orientaciones que permitan dar sustento a la construcción de conocimiento en este campo particular.

La asignatura Estudios Sociales forma parte del conjunto de materias que integran el currículo escolar de Costa Rica y de otros países del mundo, y además constituye un campo o disciplina académica específica. Una característica de la disciplina de los Estudios Sociales, es la ambigua delimitación de su campo y objeto de estudio, al punto de que se ha tomado en algunos casos como una disciplina que puede abarcar casi cualquier tema, abordando desde aspectos geológicos hasta temáticas como la seguridad vial (Costa Rica. Ministerio de Educación Publica [MEP], 2008). De ello, entre otros factores, se derivan algunas dificultades en el establecimiento y delimitación de sus líneas investigativas y sobre todo en sus fundamentos epistemológicos. Aportar algunos elementos a la discusión en torno a los probables caminos de una epistemología de la investigación en la enseñanza de los Estudios Sociales es el objetivo central de este artículo.

Las Ciencias Sociales en general, a pesar de los constantes debates en torno a cuestiones metodológicas, epistémicas o teóricas, poseen campos relativamente bien definidos en lo que se refiere a sus perspectivas de análisis y a la construcción de sus objetos de estudio y de investigación. La investigación en Sociología, Historia o Antropología goza de una perspectiva y pertinencia basada en un lenguaje común que es transversal a la comunidad académica que se ocupa de estas áreas del conocimiento, y al reconocimiento de objetos de estudio que les son propios.

De esta manera, la mayoría de las Ciencias Sociales ha logrado desarrollarse sobre cimientos teórico-metodológicos y sobre todo epistemológicos diversos pero sólidos, que posibilitan la constante construcción 
de nuevos conocimientos y la reelaboración de sus categorías conceptuales. No es posible afirmar lo mismo en el campo de la enseñanza de los Estudios Sociales, puesto que el desarrollo de la disciplina (a pesar de los múltiples, diversos y valiosos aportes que se han brindado mediante procesos investigativos en este campo) se ha dado de una manera desarticulada, debido a la multiplicidad de áreas del conocimiento que intervienen en ella y la constituyen. Además de una dificultad en la clarificación epistémica y teórico-metodológica que existe en torno a la definición del objeto de estudio propio de la disciplina. Esto se puede evidenciar revisando la discusión o la poca discusión existente en el campo y la versatilidad del área para absorber las más disimiles temáticas, las cuales en muchos casos, tienen poco que ver entre sí (Solano, 2006).

Por lo tanto, en este artículo se reflexiona sobre el problema de la conceptualización de la enseñanza de los Estudios Sociales, analizando su origen, sus características y su problemática, con el fin observar las causas de las dificultades que existen en la delimitación y establecimiento de líneas epistemológicas y teórico-metodológicas que puedan organizar el campo de investigación de la disciplina.

Además, se esbozan algunas orientaciones, sobre todo de carácter epistemológico para la investigación y la construcción de conocimiento en el área de la enseñanza de los Estudios Sociales. Esto con el objetivo primordial de brindarle a la disciplina su estatus académico como un área del conocimiento que se mueve entre los terrenos de las Ciencias Sociales y de las ciencias pedagógicas.

\section{La enseñanza de los Estudios Sociales y la didáctica de las Ciencias Sociales: un problema conceptual}

Tradicionalmente se ha considerado que la enseñanza de los Estudios Sociales es simplemente una asignatura más dentro del currículo escolar. Sin embargo, es necesario además de ello reconocer y reivindicar su carácter de disciplina académica; es decir, ubicar la enseñanza de los Estudios Sociales como una rama del conocimiento con un objeto de estudio definido y particular, con una serie de principios que le permitan a la comunidad académica que se ocupa de esta área construir nuevos conocimientos, todo ello a pesar de su singular ubicación entre las Ciencias Sociales y las Ciencias de la Educación.

No obstante, para alcanzar el reconocimiento de la enseñanza de los Estudios Sociales como una disciplina académica y como un campo autorizado para construir conocimiento válido, novedoso y pertinente mediante procesos investigativos, es necesario superar algunas problemáticas que están en la raíz de su propia definición.

Uno de los principales problemas que enfrenta la enseñanza de los Estudios Sociales para consolidarse como una disciplina académica y trascender el carácter de asignatura en sentido lato es precisamente su definición. El concepto Estudios Sociales nace en el sistema educativo estadounidense en el año de 1916, como resultado de las disposiciones adoptadas por la Comisión de la Reorganización de la Educación Secundaria (Polanco, 1984).

A partir de ese momento surgen diversas definiciones de lo que es la disciplina, sin embargo, esas múltiples definiciones tienen un factor común, el cual es su amplitud desmesurada, además de un conservadurismo implícito ligado al concepto de buen ciudadano en el marco del estructural-funcionalismo estadounidense (Torres, 1974), que posteriormente derivará en la educación ciudadana o educación cívica. Un ejemplo claro de este tipo de definiciones a las cuales se ha hecho referencia, relativas al concepto de enseñanza de los Estudios Sociales, es la siguiente:

El término Estudios Sociales se ha empleado como sinónimo de historia; como instrucción patriótica de valores; como ciencias centradas en la actividad del hombre; como las ciencias que estudian la relación 
del hombre con el hombre y con el medio ambiente; igualmente se ha identificado de acuerdo con el énfasis específico que se dé a la enseñanza, ya sean Ciencias Sociales, estudios étnicos, desarrollo moral, comprensión internacional, proceso de toma de decisiones, área de intereses sociales especializados, estudios del futuro, enseñanza sobre las armas nucleares etc. (Santamaría, Méndez y Barrantes, 2003, p. 4-5)

Tal como se evidencia en la definición que aportan estos autores, hay una perspectiva que considera la enseñanza de los Estudios Sociales como un campo que se ocupa de temas tan amplios que sus límites se vuelven difusos, abarcando elaboraciones o temas propios prácticamente de todas las Ciencias Sociales e incluso de algunas ciencias físicas como la Geología o la Meteorología. De ello se deriva que la problemática evidenciada en la definición del término se encuentra vigente y exista un debate abierto en torno al establecimiento de los límites de la disciplina. Al respecto de tal problemática, los autores antes citados indican lo siguiente:

El concepto Estudios Sociales varía de un país a otro, y aun en un mismo país, los expertos no logran ponerse de acuerdo acerca de su significación y sus alcances. (Santamaría et ál., 2003, p. 7)

Por lo tanto, una de las tesis centrales de este ensayo es que de la problemática definición conceptual de la disciplina se derivan dificultades en el establecimiento de los principios epistémicos y, por ende, de los objetos de estudio e investigación que la enseñanza de los Estudios Sociales podría abordar como parte de su campo de análisis con tal de construir nuevos conocimientos. Es decir, un problema de fondo es que de la confusa conceptualización de la enseñanza de los Estudios Sociales se deriva un problema en la delimitación de una epistemología propia. Este problema relacionado con el concepto de enseñanza de los Estudios Sociales, es bien apuntado por Salas (1999), quien lo señala de la siguiente manera:
En los ámbitos académicos universitarios, el asunto se convierte en una piedrita que molesta la tarea fácil de aquellos que, entregados a la formulación de planes de estudio, prefieren repartir créditos, cursos, horas de clase y contenidos entre las disciplinas que fluyen de la formación de educadores en Estudios Sociales. En lo que si están de acuerdo es en decir que no son una disciplina, porque no tienen objeto de estudio ni métodos propios, se descarta por completo que puedan llegar a ciencia... (p. 50-52)

Como se puede evidenciar, los argumentos planteados por Salas dan cuenta del carácter problemático con que se lidia en el campo de los Estudios Sociales, en relación a su definición, sobre todo en lo concerniente a la construcción de un objeto de estudio y unos métodos propios. Asimismo, permite observar que una de las tendencias en la disciplina es recurrir a una suerte de tecnocracia, en la que lo que importa es abordar contenidos como un fin en sí mismo.

Además de la problemática definición del concepto y del campo disciplinar de los Estudios Sociales, al ser la enseñanza de los Estudios Sociales un campo en el que intervienen variados científicos sociales, hay por lo tanto diversas perspectivas de investigación que difieren tanto en cuestiones relativas a las líneas temáticas consideradas "válidas", así como en las teorías y los métodos apropiados para acercarse a variados objetos de estudio. Es decir, no solo se carece de objeto y método propio, tal como señalaba Salas (1999), sino que además intervienen diversos campos disciplinares.

De esta problemática se deriva que la construcción de un campo de estudio y un objeto de investigación delimitado resulte una tarea relativamente difícil de lograr, puesto que, sin menospreciar los aportes que los científicos sociales de múltiples áreas podrían brindar -y efectivamente lo han hecho-, hay una tendencia a sobreponer la perspectiva de estas Ciencias Sociales, cualquiera que sea, por encima de la perspectiva de la enseñanza de los Estudios Sociales. Como resultado, se tiene trabajos de investigación que tienen un 
corte más histórico o geográfico que un carácter de investigación en el área de la enseñanza de los Estudios Sociales.

Por otra parte, generalmente se ha considerado que las Ciencias Sociales preponderantes en la enseñanza de los Estudios Sociales son la Historia, la Geografía y más tardíamente la Formación Ciudadana (Solano, 2006), sobre lo cual se podría discutir ampliamente; por ejemplo, Aisenberg (2003) indica lo siguiente:

Nuestros marcos teóricos para estudiar la enseñanza de las ciencias sociales constituyen más bien una sumatoria de recortes teóricos provenientes de diferentes disciplinas -con algunas articulaciones y/o yuxtaposiciones-, que una teoría unitaria. En consecuencia, "atrapamos" más fácilmente aspectos o recortes de nuestro objeto que al objeto en su totalidad. En virtud de la complejidad del objeto -y de nuestra formación "parcial" - es comprensible que, por ejemplo, para quienes provienen de la Historia o de la Geografía sean observables problemas relativos a los contenidos, que podrían pasar desapercibidos para profesionales provenientes del campo psicológico; y, del mismo modo, aspectos del aprendizaje claramente observables para quienes provienen de la Psicología podrían no ser registrados por otros especialistas(...). (p. 143-144)

Lo planteado por Aisemberg permite observar cómo la discusión en torno a la preponderancia de unas u otras disciplinas en el campo de la Enseñanza de las Ciencias Sociales y cómo el hecho de tener mayor o menor formación en una $u$ otra Ciencia Social puede determinar el quehacer docente que se ocupa de la Enseñanza de las Ciencias Sociales en general, y en el caso de nuestro país, de la Enseñanza de los Estudios Sociales.

Además de ello se ha considerado también, dentro de esta dispersión, que existe una gama de ciencias auxiliares que incluyen al resto de las Ciencias Sociales, las cuales en algunos casos pasan desapercibidas y no porque sean poco importantes, sino porque los profesionales en la enseñanza de los Estudios Sociales no les confieren la importancia que merecen, ya sea por falta de interés o de formación, entre otras causas, y además porque las demandas curriculares del sistema de enseñanza formal no incluyen de manera sustancial la reflexión sociológica, antropológica o económica (Hernández, 2001).

En todo caso, lo que la enseñanza de los Estudios Sociales ha tomado del conjunto de las Ciencias Sociales ha sido básicamente sus elaboraciones finales, es decir, las conclusiones a las cuales las Ciencias Sociales han llegado después de procesos investigativos. De ello resulta la inexistencia de un saber o conocimiento propio y, en consecuencia, el desarrollo de una suerte de modelo de transposición didáctica al estilo de la propuesta planteada por Chevallard (1994), en el tanto se toman las elaboraciones de las Ciencias Sociales en general y se adaptan a los espacios educativos escolares de una manera instructiva en sentido lato.

Lo que quiere decir esto es que se parte del saber de los eruditos en Ciencias Sociales y se busca adaptarlo a los espacios educativos, pues el trabajo docente que se desarrolla se nutre de los resultados de investigación en Ciencias Sociales. Lo anterior se evidencia en la forma en que se organiza el currículo escolar oficial del Ministerio de Educación (lo cual no quiere decir que todos los docentes se encuentren atados por el programa ) donde se deja poco espacio para la creación de conocimiento desde el aula. Es por ello que, en el momento de iniciar procesos de construcción de nuevo conocimiento en la enseñanza de los Estudios Sociales, se generan dificultades en la precision y delimitación tanto de los objetos de estudio, así como de los problemas de investigación (Rodríguez, 2000).

Por otra parte, en contraposición al concepto de Estudios Sociales, se encuentra el concepto y disciplina académica conocida como Didáctica de las Ciencias Sociales, que posee una connotación distinta que hasta cierto punto enriquece la formación de los estudiantes y el desempeño de los docentes que se ocupan de esta área (Domínguez, 2004). La Didáctica de las Ciencias Sociales tiene una definición un 
tanto más acotada y menos difusa, y ha sido una asignatura desarrollada en varios países en el nivel de la educación general, con un desarrollo notable en Europa (donde un referente importante lo constituye el caso de España), y a pesar de que la discusión en torno a cuestiones epistemológicas y teórico-metodológicas en la Didáctica de las Ciencias Sociales es relativamente reciente, hay una cantidad destacable de reflexiones en relación a estas temáticas. ${ }^{1}$

La didáctica de las Ciencias Sociales ha sido entendida como una disciplina que si bien es un campo de encuentro entre las Ciencias Sociales y la Didáctica, posee un objeto de estudio propio y definido, el cual desde la perspectiva de Xavier Hernández, consiste en el estudio de los procesos de comunicación y enseñanza así como de recepción y aprendizaje de las Ciencias Sociales por parte de los estudiantes. En tal sentido, Hernández indica lo siguiente:

El objeto de la didáctica de las Ciencias Sociales es analizar, diseñar e investigar acerca de las técnicas de didáctica-difusión-comunicación y los procesos de comprensión/conocimiento con respecto a la historia, la geografía y la sociedad, y con respecto a los saberes que las ciencias históricas, geográficas y sociales aportan a su conocimiento. (Hernández, 2000, p. 24)

Siguiendo el planteamiento de Xavier Hernández, la Didáctica de las Ciencias Sociales es un campo disciplinar que se ocupa de analizar e investigar sobre las formas y las técnicas que podrían hacer comprensible un determinado eje temático en un contexto particular.

Además de ello, Joaquín Prats brinda una definición interesante de la didáctica de las Ciencias Sociales que permite delimitar el campo de estudio de esta disciplina con una precisión bastante aguda. Prats define la didáctica de las Ciencias Sociales como "un saber científico de carácter tecnológico, al que se une un hacer técnico" (Prats, 2003, p. 3). Prats aporta esta definición de la Didáctica de las Ciencias Sociales caracterizada por tres elementos fundamentales: un saber científico, un saber tecnológico y un hacer técnico. La considera un saber científico en el tanto se reciben y se toman de las Ciencias Sociales conceptos, teorías y conocimientos resultantes de procesos investigativos propios de ellas. Además, se considera también un saber tecnológico ya que el conocimiento que se produce en las disciplinas de las Ciencias Sociales se lleva a la práctica en espacios de enseñanza y aprendizaje de una manera sistemática y dirigida, que tiene por objetivo que los estudiantes conozcan y desarrollen métodos y experiencias propias de las Ciencias Sociales. Y en última instancia, es además un hacer técnico en el que la actividad docente asume una serie de normas, reglas y protocolos para obtener resultados de aprendizaje mediante la aplicación de las técnicas y metodologías propias de las ciencias sociales.

Por otra parte, el objetivo de la Didáctica de las Ciencias Sociales no es meramente instructivo o de transposición didáctica -tal como se desprende de las definiciones que tradicionalmente se han elaborado sobre la enseñanza de los Estudios Sociales, y del origen de los Estudios Sociales en el marco del estructural-funcionalismo estadounidense, de carácter claramente conservador-, sino que persigue que los estudiantes puedan aprehender métodos propios de las Ciencias Sociales con tal de que desarrollen capacidades investigativas y por lo tanto puedan construir su propio conocimiento en el área de las Ciencias Sociales. Así como lo plantea Joaquín Prats, los contenidos propios de una didáctica de las Ciencias Sociales poseen un carácter de simulación de situaciones y procesos investigativos característicos de las Ciencias Sociales:

Los contenidos [de la didáctica de las Ciencias Sociales] atienden a la importancia de la simulación. Es decir, crean escenarios de copia o simulación de situaciones o comportamientos de investigación en las Ciencias Sociales de modo creativo. La simulación será a la vez método y contenido específico (...). (Prats, 2000, p. 15) 
Desde la perspectiva de Prats, la simulación de los procesos de investigación debe ser un contenido de la enseñanza de las Ciencias Sociales; sin embargo, indica también que para ello es necesario que el profesorado conozca los protocolos y el modus operandis de al menos una de las Ciencias Sociales, puesto que, como bien lo argumenta el autor, la simulación de estos procesos estaría mal desarrollada en el tanto no se conozca bien lo que se simula. Por ello, las formas como se produce el conocimiento en las Ciencias Sociales debe ser parte esencial en la formación de los docentes, sobrepasando así la mera aprehensión y transposición didáctica de contenidos.

\section{Epistemología para la investigación en la disciplina de la enseñanza de los Estudios Sociales}

Con tal de delimitar una epistemología propia para la investigación en la enseñanza de los Estudios Sociales, se considera que se debe iniciar por abandonar las definiciones difusas que tradicionalmente se han desarrollado en torno al concepto de Enseñanza de los Estudios Sociales y acercarse más a los aportes que se han brindado desde la tradición de la didáctica de las Ciencias Sociales. Esto por el carácter un tanto imprecisas de esas definiciones tradicionales y por el hecho de poseer una tendencia muy fuerte hacia la transposición didáctica, puesto que ambos son aspectos que generan problemas a la hora de delimitar el campo de estudio de la disciplina y las líneas de investigación del área.

Uno de los aspectos centrales para definir los caminos de una epistemología para la investigación en la disciplina de la enseñanza de los Estudios Sociales es retomar la tarea de definir un objeto de estudio claro a la hora de iniciar procesos investi- gativos. Tal objeto, dado el carácter de esta didáctica específica, debe estar en función de las líneas generales de la epistemología de las Ciencias Sociales, así como del mejoramiento de los procesos de enseñanza y aprendizaje de tales ciencias en un sentido amplio (es decir, tanto en lo referente a los conceptos, las teorías, las metodologías, y los conocimientos propios de las Ciencias Sociales, al igual que en lo concerniente a los protocolos o los habitus académicos, intelectuales y de investigación de las principales Ciencias Sociales).

Esto requiere que la formación de los docentes en el campo de la enseñanza de los Estudios Sociales incluya la formación rigurosa en los hábitos académicos de al menos una de las Ciencias Sociales, y nunca poner el énfasis tan solo en el conocimiento de los resultados finales que se obtienen de los procesos de investigación de las Ciencias Sociales con tal de llevarlos o reproducirlos en las aulas. En otras palabras, hay que dejar de reproducir saberes y empezar a producir conocimiento de carácter científico-social desde las aulas (Sobejano, 2000).

Como se ha sugerido, con tal de conferirle el estatus académico e intelectual que merece a la disciplina de la enseñanza de los Estudios Sociales, se debe asumir, discutir y reconstruir una epistemología propia de las Ciencias Sociales, adecuándola a las particularidades de esta didáctica específica. Por lo tanto, uno de los pasos primordiales, y quizá el punto de arranque, es el abandono de toda noción previa en el momento de construcción de objetos de estudio que tengan validez como objetos de investigación.

En este sentido se recuerda una máxima de las Ciencias Sociales aportada por Emile Durkheim, quien no solo es uno de los fundadores de la sociología como disciplina científica, sino que además se desempeñó durante varios años como profesor titular de la Cátedra de Pedagogía en la Universidad de la Sorbona en Francia, el principio de abandonar sistemáticamente 
las nociones preconcebidas (Durkheim, 2001). Este es uno de e los planteamientos epistemológicos fundamentales de Durkheim, pues destaca el axioma que indica que es imprescindible alejarse del mundo de la doxa, de la tiranía de las concepciones previas sobre un determinado hecho o fenómeno. Durkheim plantea categóricamente que es "preciso evitar sistemáticamente todas las prenociones" (Durkheim, 2001, p. 44).

Tomando como referencia este planteamiento, la implicacion para los procesos investigativos en la enseñanza de los Estudios Sociales resulta en que estos deben necesariamente construir sus objetos de investigación alejándose lo más posible de los juicios previos y de las afirmaciones carentes de fundamentacion teorico-empírica, propias del mundo de la opinión.

En la educación en general, y particularmente en la enseñanza de los Estudios Sociales, resulta sencillo dejarse llevar por el mundo de la doxa (Wacquant, 2006), es decir del sentido común, y lanzar afirmaciones que carecen de fundamentacion teórica o al menos empírica, constituyendo tan solo opiniones. De ello se deriva que lejos de construir verdaderos problemas de investigacion en el campo de la enseñanza de los Estudios Sociales, apenas se enuncien aparentes problemas sociales, educativos o didácticos, de los cuales en muchos casos se carece de evidencia que los confirme.

Por lo tanto, cualquier problema de investigación u objeto de estudio propio de la enseñanza de los Estudios Sociales debe basarse en una fundamentacion empírica o teórica que justifique la elaboración o construcción de los diseños investigativos. No basta con que se conjeture la existencia de algún problema en la enseñanza de los Estudios Sociales, hay que dar el salto a la problematizacion científica, es necesario efectuar un proceso de ruptura con las nociones espontaneas:

La investigacion cientifica se organiza de hecho en torno a objetos construidos que no tienen nada en comun con aquellas unidades delimitadas por la percepcion ingenua. (Bourdieu, Chamboredon, Passeron, 2003, p. 53)

Se requiere, por lo tanto, aportar evidencia empírica y teórica que justifique la eleccion o delimitación de un determinado objeto de investigación. ${ }^{2}$ Esto es necesario en el tanto de que la enseñanza de los Estudios Sociales es una disciplina que se enmarca en las Ciencias Sociales, cuyos objetos de estudio, al implicar al sujeto, son suceptibles de dejarse marcar por juicios o apreciaciones subjetivas o aceptadas socialmente, aunque no correspondan a la realidad social.

Hay que tener en cuenta que no existen objetos dados, con carácter ontológico sino que los objetos de investigación resultan de la construccion del investigador. Parafraseando a Max Weber, no son las relaciones reales entre las cosas, sino las relaciones conceptuales entre problemas (Weber, 1971) las que se encargan de delimitar objetos de estudio o investigacion científica. Esas relaciones conceptuales son producto de la elaboración y disertación teórica en torno a los objetos abarcables, sobre lo cual, Bourdieu, Chamboredon y Passeron afirma que:

Un objeto de investigación, por más parcial y parcelario que sea, no puede ser definido y construido sino en función de una problemática teórica que permita someter a un sistematico examen todos los aspectos de la realidad puestos en relación por los problemas que les son planteados. (Bourdieu et ál. 2003, p. 54)

El objeto de investigación no existe per se, lo que existe es la realidad adjetivada, es decir, social, educativa, didactica o de otra índole, y la realidad está desordenada, hay que ordenarla conceptualmente, es esa la función del científico social (Berger y Luckmann, 1991). Por lo tanto, igualmente los objetos de investigación en la enseñanza de los Estudios Sociales deben partir de una de una problematizacion teórica que les dé un estatus de fenomenos "dignos de ser estudiados" y, además, su delimitación debe aprovechar evidencia empírica, constatable, que les brinde justificación. 
El objeto se alcanza mediante la ruptura con la realidad bruta, luego se construye y posteriormente se comprueba (Bourdieu et ál. 2003) para volver o reoperar sobre la base teórico-empírica que le dio origen.

Igualmente, en la disciplina de la Enseñanza de los Estudios Sociales, asícomo en el resto de las Ciencias de la Educación, el objeto debe ser una construcción del investigador. Claramente, esta construcción no es ni arbitraria ni unilateral, si no que debe estar basada en la problematización teórica y la evidencia empírica que la sustente, lo cual implica una ruptura con el orden del sentido común, y por lo tanto el alcance del hecho científico, que es previo a cualquier comprobacion o aplicación de los aparatos metodológicos construidos en función del objeto y cuya finalidad última es captarlo.

Por último, es necesario referirse al tema del compromiso del investigador con su objeto, el cual es otro problema (de considerables implicaciones epistemológicas) que se manisiesta en el conjunto de las Ciencias Sociales y es muy comun igualmente en la investigacion en la enseñanza de los Estudios Sociales. Hay en la investigacion educativa una fuerte tendencia -muy respetable e incluso valiosa- a asumir un compromiso fuerte con los temas que se investigan, debido al carácter de intelecutal transformador que se le ha asignado al educador. Igualmente el profesor de Estudios Sociales asume un papel de alto compromiso debido a su función de enseñar las ciencias que se ocupan de la vida social, y por lo tanto, que tienen entre otras funciones, denunciar la dominación, la explotación y las inequidades del más variado tipo.

A pesar de ello es importante tener en cuenta que si bien la subjetividad no se puede cancelar a la hora de emprender procesos investigativos, tal subjetividad debe ser al menos explicitada, y en el mejor de los casos vigilada.

La educación y la enseñanza de los Estudios Sociales, como cualquier otro sector de la realidad social, posee un elemento que no puede ser ignorado, el cual es el hecho de que la relacion sujeto-objeto no puede en ningún caso ser totalmente separada, ya que tanto el sujeto cognoscente como el objeto cognosible forman parte de una estructura social. En tal sentido, Lucien Goldmann sostiene que:

En efecto, toda realidad social se halla constituida al mismo tiempo por hechos materiales y por hechos intelectuales y afectivos, que a su vez estructuran la conciencia del investigador y que lógicamente comportan evaluaciones. He aquí porque nos parece imposible un estudio rigurosamente objetivo de la sociedad. (Goldmann, 1979, p. 74)

Por lo tanto, la aspirada objetividad es un asunto variable en función de la cercanía, la familiaridad y el compromiso que el investigador posea respecto de su objeto, lo cual supone la necesidad de vigilar la subjetividad del investigador, explicitando los grados de vinculación, con el fin de evitar en la medida de lo posible que la subjetividad se superponga sobre el proceso investigativo, sobre lo cual Goldmann señala que:

...es menester destacar lo más claramente posible el grado específico de identidad entre el sujeto y el objeto, y por lo mismo el grado de objetividad que puede alcanzar la investigacion. (Goldmann, 1979, p. 74)

Una derivación de la problemática del grado de familiaridad o vinculación a espacios educativos puede no solo modificar en mucho los resultados de la investigación, sino que incluso podría, además, exacerbar el criterio de la conveniencia, utilizándolo no para selccionar a los sujetos de investigación, o idealmente, la muestra, si no para delinear objetos de investigación. Sobre esta problemática, el siguiente pronunciamiento aclara:

Frente a algunas investigaciones concebidas en función de las necesidades de la causa lógica o metodológica, no puede si no evocarse, con Abraham Kaplan, la conducta de un borracho que, habiendo perdido la llave de su casa, la busca sin embargo con obstinación, bajo la luz de un farol, ya que alega que allí se ve mejor. (Bourdieu et ál., 2003, p. 21) 
No queda más, por lo tanto, que controlar el grado de vinculación con una realidad social o bien con los planteamientos o corrientes ideológicas asumidas, pues de otro modo se podría terminar falseando el proceso de investigación, tomando caminos que no solo son sesgados sino que además resultan facilistas.

En última instancia, parafraseando a Norberth Elías, en favor de la construcción de objetos de investigación delineados no por las simples aspiraciones personales o por el compromiso con una u otra realidad social, habría que decir que los científicos sociales no pueden solucionar la tensión entre su papel de sujetos inmersos y vinculados a las realidades que se estudian, renunciando a su rol de seres humanos en favor de su rol de investigador, pues más bien, su compromiso forma parte de las condiciones necesarias para comprender el problema que se intenta solucionar científicamente (Elías, 1983). Sin embargo, ese compromiso debe ser controlado y explícito, asumiendo mesuradamente la relación con el objeto de estudio, por una sana cuestión de honestidad intelectual.

\section{Orientaciones para la investigación en la Enseñanza de los Estudios Sociales}

Después de lo expuesto en el apartado anterior, se cree firmemente que lo esencial para los procesos de investigación en la enseñanza de los Estudios Sociales es sobre todo tener claro el proceso de construcción del objeto de estudio, tomando como base la epistemología fundamental de las Ciencias Sociales, esto en el sentido de que la enseñanza de las Ciencias Sociales forma parte integral de ese conjunto mayor de disciplinas científicas.

Por otro lado, existen tantas posibilidades de objetos de investigación, como sujetos y enfoques existan. Se sostiene la tesis de que las líneas temáticas en la investigación de la enseñanza de los Estudios Sociales es inagotable en tanto que la realidad social, pedagógica, didáctica y educativa es también inagotable: el sujeto capta lo real y lo representa mediante procesos racionales, pero el sujeto no tiene la posibilidad de agotar lo real, ya que la totalidad de lo real es inabarcable e irreproducible.

En tal sentido, está claro además que la construcción de nuevos conocimientos en el área de la enseñanza de los Estudios Sociales mediante procesos investigativos no es, ni pretende ser un camino mediante el cual surja una suerte de conocimientos de pretendida validez universal; sin embargo, esos conocimientos sí tienen el carácter de saber científico en tanto sean obtenidos mediante la aplicación del método científico, sobre lo cual Joaquim Prats afirma lo siguiente:

Soy consciente que no es objetivo de la investigación en Didáctica de las Ciencias Sociales establecer leyes universales, ni tan solo generales, pero sí que es posible establecer normas que, delimitando y expresando el campo de sus condiciones contextuales, pueden tener un cierto valor de generalización. Por lo tanto puede afirmarse que los resultados de la investigación en este ámbito pueden alcanzar el estatus de conocimiento científico. (Prats, 2003, p. 2)

No obstante, a pesar de tal reconocimiento, es importante tener en cuenta que existen valiosos intentos por establecer líneas de investigación en la didáctica de las Ciencias Sociales y, por ende, en la enseñanza de los Estudios Sociales. Es importante aclarar que son líneas de investigación, lo cual no implica necesariamente que se propongan temas de investigación, pues en investigación no existen temáticas apropiadas, lo que existen son tratamientos novedosos, creativos, y valiosos sobre diversos temas. Por lo tanto, referirse a temáticas apropiadas sería delimitar e inhibir demasiado la capacidad creativa del investigador. Por ejemplo, el mismo Prats en el artículo titulado precisamente Líneas de investigación en didáctica de las Ciencias Sociales (Prats, 2003) propone algunos 
núcleos investigativos, los cuales advierte son apenas orientaciones inacabadas y con un carácter provisorio. Tales campos de investigación son los siguientes:

- Diseño y desarrollo curricular en sus diversas etapas, áreas y disciplinas educativas.

- Construcción de conceptos y elementos que centren el contenido relacional y polivalente de la Didáctica de las Ciencias Sociales.

- Estudios sobre comportamiento y desarrollo de la profesionalidad docente en lo referente a la enseñanza de la Historia, Geografía y Ciencias Sociales.

- Investigaciones ligadas a las concepciones de la Historia y la Geografía $\mathrm{u}$ otras Ciencias Sociales entre el alumnado, y la evaluación de los aprendizajes.

Investigaciones sobre la didáctica del patrimonio.

Estas líneas de investigación poseen el valor de constituir un intento de sistematización coherente de los caminos de la investigación en la Didáctica de las Ciencias Sociales, y por lo tanto su consiguiente utilidad para la enseñanza de los Estudios Sociales. Resultan también sumamente útiles para conocer cuáles son algunas de las principales tendencias investigativas en la didáctica de las Ciencias Sociales a nivel internacional e incluso para pensar sobre su viabilidad con tal de ser asumidas en el ámbito nacional.

Cada una de estas líneas de investigación ha sido desagregada por Prats con tal de delinear algunos ejes temáticos posibles, los cuales poseen la principal característica de constituir temas de gran interés que permiten la construcción y acumulación de conocimiento válido y contrastable, como cualquier campo o disciplina científica. No obstante, se retoma el aforismo que indica que la unidad está en el objeto y la diversidad en el enfoque; contrario a ello, partiendo de los planteamientos de Max Weber, se considera que existen igualmente objetos y enfoques diversos en tanto los objetos son construcciones del sujeto delineados mediante las relaciones conceptuales entre problemas y no simplemente mediante las relaciones reales ente las cosas, como es propio del conocimiento del sentido común.

De lo anterior se concluye que hay una gran posibilidad de ejes temáticos plausibles, los cuales sin embargo deben tener en consideración sobre todo la necesidad de explorar aspectos que mejoren tanto la compresión de los procesos de enseñanza y aprendizaje de las Ciencias Sociales, así como los procesos mismos, y tomen en cuenta todos los elementos de la situación educativa.

\section{Consideraciones finales}

Es importante tener en cuenta la necesidad de realizar o establecer un diálogo constante entre las categorías conceptuales de la Didáctica de las Ciencias Sociales y la Enseñanza de los Estudios Sociales, sobre todo con el objetivo de retomar la importancia de la precisión y clarificación conceptual, lo cual tiene como finalidad delimitar claramente los objetos de estudio de los que la investigación en la enseñanza de los Estudios Sociales podría eventualmente ocuparse.

Un paso importante también es ahondar en la discusión sobre la posibilidad de demarcar con mayor precisión el concepto de "Enseñanza de los Estudios Sociales", el cual, como se ha expuesto, ha sido sujeto de definiciones relativamente vagas, que tienen implicaciones tanto en la práctica profesional de los docentes que se ocupan del área, así como en los procesos de aprendizaje que se desarrollan en las aulas, (y por lo tanto, en los procesos investigativos de la disciplina).

Para ello se considera que resultaría muy útil tratar de alejarse de los modelos de transposición didáctica que han caracterizado a esta disciplina y empezar 
a construir conocimiento desde este campo específico, evitando quedarse únicamente en los conocimientos elaborados por las Ciencias Sociales mediante sus procesos investigativos.

Por otro lado, es necesario alcanzar una epistemología concordataria para los profesionales del área, una epistemología que se enmarque en las líneas generales de las Ciencias Sociales y que tenga como principal objetivo la clarificación de los procesos que permitan conquistar objetos de investigación válidos, pertinentes y que admitan acumular conocimiento con el fin de ser contrastado, discutido y superado, como en cualquier otro campo científico.

Se cree que resulta esencial indicar que las posibilidades de alcanzar objetos de investigación diversos y multi-determinados son inagotables, y de validez para la comunidad académica que gira en torno a la enseñanza de las Ciencias Sociales. Existen tantos objetos como sujetos con posibilidad de construirlos. Es por ello que se concluye diciendo que no hay temas imposibles, siempre y cuando reúnan una serie de requerimientos propios de esta didáctica específica y de las Ciencias Sociales en general.

En términos generales, la trascendencia y reconocimiento de los Estudios Sociales como disciplina académica, en buena medida está en función de la capacidad que se tenga de generar conocimiento novedoso y producir un cúmulo de conocimientos, métodos, y reflexiones teóricas propias, y compartidas por quienes se dedican a esta disciplina.

Es en este sentido, que la validez de los Estudios Sociales, como disciplina académica, podría ponerse de manifiesto, pues podría aportar a los estudiantes, y a la sociedad en general, mediante las herramientas conceptuales que brindan las Ciencias Sociales, los recursos para enfrentarse eficazmente a los retos que plantean las sociedades contemporáneas, caracterizadas por los flujos maximizados de información, y la complejidad de los sistemas sociales, jurídicos y económicos que se han estructurado paulatinamente.

\section{Notas}

1 Existe una bibliografía abundante en lo relativo al tema de la Didáctica de las Ciencias Sociales, de la cual, tan solo por citar algunas obras se indican las siguientes:

Aisemberg, B. (1994). Didáctica de las Ciencias Sociales. Educador: Paidós.

Prats, J. (2000). Los Caminos de la didáctica de las ciencias sociales. Madrid: Graó.

Prats, J. (2003). Líneas de investigación en didáctica de las ciencias sociales. Historia \& Ensino. Revista do laboratório de Ensino de Históriória/ UEL, Vol. 9.1-24.

Sobejano, M. J. (2000).Concepto fundamento y elementos del currículo en historia. Madrid: UNED. Hernández, X. (2001). Diseños curriculares en ciencias sociales. España: Editorial GRAO.

2 En el campo de la sociología, Pierre Boudieu, Chamboredon, y Passeron en su célebre obra titulada El oficio del sociólogo planteaban la necesidad de dar el salto de los problemas sociales a los problemas sociológicos. Este principio ha sido asumido por la comunidad académica de las Ciencias Sociales y es necesario considerarlo y tenerlo en cuenta en el área de la educación y de la enseñanza de los estudios sociales. Ello implica que no es suficiente suponer la existencia de un problema de variada índole, puesto que resulta imprescindible construirlo intelectualmente, tratando de brindar la evidencia empírica necesaria que lo fundamente, y delinearlo teóricamente con tal de elaborar un objeto de estudio y un problema de investigación de carácter científico.

\section{Referencias bibliográficas}

Aisenberg, B. (2003, Abril). Didáctica de las Ciencias Sociales. ¿Desde qué teorías estudiamos la enseñanza? Revista de Teoría y Didáctica de las Ciencias Sociales 003(3), 136-183. Recuperado de http://www.saber.ula. ve/handle/123456789/23916

Berger, P. y Luckmann, T. (1991). La construcción social de la realidad. España: Editorial Amorrortu. 
Bourdieu, P., Chamboredon, J. C. y Passeron, J. C. (2003). El oficio del sociólogo. (24ª ed.). México: Siglo XXI editores.

Chevallard, Y. (1994). Les processus de transposition didactique et leur théorisation. En G. Arsac, Y. Chevallard, J.-L. Martinand y A. Tiberghien (Coords.). La transposition didactique à l'épreuve (pp. 135-180). Grenoble: La Pensée sauvage.

Costa Rica. Ministerio de Educación Pública. (2008). Programas de Educación Cívica. San José, Costa Rica: Autor. Recuperado de http:// www.procesos.org/Descarga/ Cuadernos/CT\%202009-01\%20 Manual\%20de\%20Referencia\%20 para\%20Implementacion.pdf

Domínguez, M. C. (2004). Didáctica de las ciencias sociales para primaria. España: Pearson Educación.

Durkheim, E. (2001). Las reglas del método sociológico. México: Coyoacán: Fondo de Cultura Económica.

Elías, N. (1983). Compromiso y distanciamiento. Ensayos de sociología del conocimiento. Barcelona: Ediciones Península.

Goldmann, L. (1979). Epistemología de las ciencias del hombre. Buenos Aires: Paidós.

Hernández, F. X. (2000). Epistemología y diversidad estratágica en la didáctica de las ciencias sociales. Iber: Didáctica de las ciencias sociales, geografía e historia, 24, 19-32.

Hernández, X. (2001). Diseños curriculares en ciencias sociales. España: Editorial GRAO.
Polanco, M. E. (1984). Didáctica de los estudios sociales. (Colección E.F.A. de textos universitarios). San José: Fernández-Arce Editores.

Prats, J. (2000). Los caminos de la didactica de las ciencias sociales. Madrid: Editorial Graó.

Prats, J. (2003). Líneas de investigación en didáctica de las ciencias sociales. Historia \& Ensino. Revista do laboratório de Ensino de Históriória/ UEL , Vol. 9, 1-24.

Rodríguez, F. (2000). La elaboración del conocimiento geográfico escolar: ¿de la ciencia geográfica a la geografía que se enseña y viceversa? En J. Prats. Los Caminos de la didáctica de las ciencias sociales (pp. 107 -118). Madrid: Editorial Graó.

Salas, J. A. (1999). La incorporación de los estudios sociales como materia de estudio escolar en Costa Rica y la necesidad de su re-conceptualización de cara a los desafíos para la educación a inicios del siglo XXI. Perspectivas. Revista de Investigación, teoría y didáctica de los estudios sociales. Año 2, Vol. 2, 49-57.

Santamaría, M. A. [Coord.], Méndez, N. y Barrantes, F. (2003). La enseñanza de los estudios sociales en I y II ciclos. Antología. San José: UNED.

Sobejano, M.J. (2000). Concepto fundamento $y$ elementos del currículo en historia. Madrid: EUNED.

Solano, E.(2006,Mayo-Agosto). ¿Enseñamos Estudios Sociales o Historia? Revista Actualidades Investigativas en Educación, 6(2), 1-20.

Torres, E. (1974). Introducción al pensamiento sociológico: Marx, 
Weber, Durkheim, Parsons. Costa Rica: Editorial universitaria centroamericana. Pensamiento crítico y disolución de la

Wacquant, L. (2006, Enero-Junio). Weber,M.(1971).Sobrelateoríadelasciencias

doxa: Entrevista con Loïc Wacquant. ANTIPODA, Número 2, 43-50. Universidad de los Andes, Colombia. sociales. Barcelona: Península. 